\title{
PVC Gel based Artificial Muscles: Characterizations and Actuation Modular Constructions
}

\author{
Yi LI ${ }^{* 1}$ and Minoru HASHIMOTO*1 ${ }^{* 1}$ Member, IEEE \\ ${ }^{* 1}$ Faculty of Textile Science and Technology, Shinshu University, 3-15-1 Tokida, Ueda, \\ Nagano 386-8567, Japan. \\ Correspondence to: Y. Li (liki@shinshu-u.ac.jp)
}

\begin{abstract}
Polymer materials based artificial muscles have the properties of being soft, lightweight and flexible which are similar to the nature muscular actuators. In our previous study, we have developed a contraction type artificial muscle based on plasticized poly vinyl chloride (PVC) gel and meshed electrodes. And we have improved the characteristics to make it close to the level of natural muscle. It has many positive characteristics, such as stable actuation in the air, high output, notable response rate and low power consumption. So a wide application is expected. However, for practical applications, it is necessary to consider some specific criteria, such as performance criteria and structural criteria. In this study, we introduced the most updated properties of PVC gel artificial muscles and proposed three types of mechanical actuation modular constructions for making the PVC gel artificial muscle as a robust actuation device for robotics and mechatronics. And we tested a prototype to examine the effectiveness of the proposed modules. Finally, an analytical model for the static characteristics of PVC gel artificial muscles at different applied voltages was derived and showed good agreement with experimental results measured by a prototype of modules.
\end{abstract}

Key Words : Polymer material, Artificial muscle, PVC gel, Soft actuator, Modular construction

\section{Introduction}

The most common actuators are combustion engines, electric motors and piezoelectrics. Combustion engines have high efficiency when operated continuously but not suitable for the intermittent motion like walking robots. Electric motors have high accuracy but low efficiency in torque to mass compared to biological muscles, which makes them very bulky for robotic, medical and fluidic applications. Piezoceramics have very high power densities but with a small strain of $0.1 \%$ which makes massive mechanical amplification necessary when significant displacements are required. These types of traditional actuators are not ideal for the applications in the mechatronics or biomimetics, such as rehabilitation devices, power assist suits and insect type micro robots. For these applications, the actuators that have the similar characteristics of biological muscles of light in weight, high strain and stress, soft and low noise are desirable.

Polymer material based artificial muscles have attracted great attention these years $[1,2]$. A variety of electronic, ionic, and photoactivated artificial muscles are proposed that exhibit large strain in length and volume, high response rate, and high output power, which is the primary similarity with muscle [3, 4]. The pneumatic artificial muscles (PAMs) [5, 6] have the advantages of high force to weight ratio, lightweight, low cost and flexibility. But generally a heavy and noisy energy resource (air or gas) equipment is needed. Shape memory polymers (SMPs) $[7,8]$ can be developed into multifunctional materials actuated by various methods, such as thermal-induced, electro-activated and magnetic-actuated SMPs. They can achieve a high strain and stress but a low response rate (sec to $\mathrm{min}$ ). On the other hand, electro active polymers (EAPs) which exhibit large strain in response to electrical stimulation, are human made actuators that most closely emulate muscles. The most attractive feature of EAPs is their ability to emulate biological muscles offering resilience, toughness, large actuation strain and inherent vibration damping. Ionic polymer-metal composites (IPMCs) [9,
10] are one type of EAP actuators that create a large bending motion under relatively low input voltage (1 3V). However, the response rate is not notable and there is a need to maintain their wetness. Dielectric elastomer actuators (DEAs) are one of the most studied EAP actuators and numerous applications are being developed including electroactive fluid pumps, conformal skins for Braille screens and insect-like robots [11-13]. However, the challenge for this type of actuators is their use of a high voltage $(>1 \mathrm{kV})$ due to the high electric fields that are needed $(\sim 100 \mathrm{~V} / \mu \mathrm{m})$.

Among all the candidates of artificial muscles, PVC gel based artificial muscles showed a great potential for practical applications. Hirai et al. [14] found the bending deformation of PVC gel under an electric field. However, to emulate nature's muscle, we think it is necessary to create a contraction and expansion movement. Based on the unique phenomenon and mechanism of PVC gel at the side of anode of electrode, we have developed a contraction and expansion type PVC gel artificial muscle, using the meshed anode and foil cathode [15]. And we evaluated and improved its characteristics [16, 17], modeled it as a control element [18]. Also some applications, such as a motor brake [19] and walking assist gel spats [20] are implemented so far. The PVC gel artificial muscle has the advantages of stable movement in the air with a large strain and an output stress with a high response rate and stability on thermal influence. And a medium input electric field $(\sim 20 \mathrm{~V} / \mu \mathrm{m})$ and a low level of power consumption $(\sim 5 \mu \mathrm{W} / \mu \mathrm{m})$ which also show the reasonability for practical applications. Furthermore, the durability (cycle life) under a continuous electric field driven is also confirmed sufficient for the practical applications, recently.

Since each actuator is defined according to the physical theory on which its legitimacy is founded, a general theory of actuators does not exist. Each actuator has its own composition, mechanism, motion type and mechanical structure. As an alternative among the artificial muscles or the traditional 
actuators in practical applications, it is necessary to consider some specific criteria, such as performance criteria and structural criteria. In this study, we introduced the composition, mechanism and present the most updated properties of PVC gel artificial muscles. And considering one step for various practical applications of PVC gel artificial muscles in the future, we proposed the actuation modular constructions with different functions to make the PVC gel artificial muscle as an useful soft actuator in practice. And we gave an example to show the effectiveness of the proposed modules and developed a model of static characteristics of PVC gel artificial muscles based on the experimental data measured by a prototype of mudules.

This paper is organized as follows: The composition and mechanism of PVC gels are introduced in Section 2. The structure and characteristics of PVC gel artificial muscles are described in Section 3, and the actuation modular constructions are proposed in Section 4. The fabricated module unit and experimental results are presented in Section 5. Finally, some discussions and conclusions are addressed in Section 6 and Section 7, respectively.

\section{Composition and deformation mechanism of PVC gels}

\subsection{Materials and fabrication of PVC gels}

Plasticized PVC gel was made from commercial PVC powder (degree of polymerization $=3200$ ), dibutyl adipate (DBA) plasticizer and tetrahydrofuran (THF) solvent with different weight ratios. The mixed solution was cast in a petri dish and the THF was evaporated at a constant temperature of $20^{\circ} \mathrm{C}$ for two days to get a thin film gel, as one kind of soft dielectric elastomer with high transparency (Fig. 1). Stiffness of PVC gel can be adjusted by the weight ratio of PVC to DBA. The lower stiffness was achieved with the higher weight ratio of DBA. In this study, the weight ratio of PVC and DBA was adjusted to 1:4.

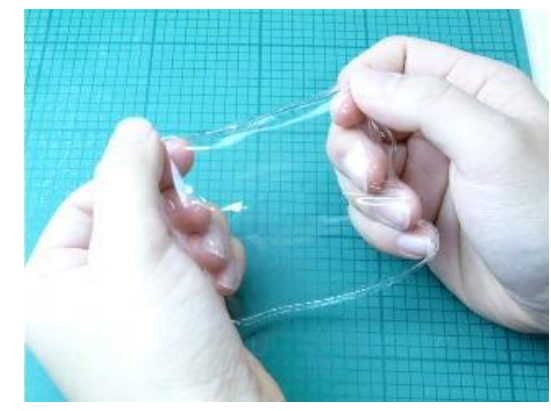

Fig. 1. A plasticized PVC gel film.

\subsection{Deformation mechanism of PVC gels}

As shown in Fig. 2, when PVC gel is sandwiched between electrodes, creep deformation takes place between the gel and the anode when the electric field is charged. And with the electric field is turned off, the gel returns to its original shape quickly by its own elasticity. A phenomenon that an accumulation of negative charges on the gel surface near the anode was confirmed by space-charge measurement [21]. It was also found that PVC gels were separated to two layers obviously after applied an electrical field, of which the layer near the anode was thinner and softer than other layer due to the transfer of plasticizer to the anode [22]. Therefore, we believe that when an electric field is turned on, electrons are injected from the cathode into the gel, and migrate toward the anode. Due to the Maxwell force, the PVC gel will be deformed asymmetrically and results in creep deformation along the anode. Based on this hypothesis, we simulated the deformation of PVC gel by finite element method (FEM) and got the same creep deformation [23].
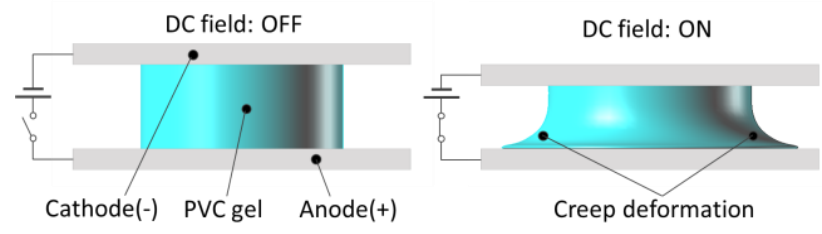

Fig. 2. Deformation mechanism of PVC gel.

\section{Structure and properties of PVC gel artificial muscles}

\subsection{Structure of PVC gel artificial muscles}

Considering the unique creep deformation near the anode, we proposed the structure of a contraction and expansion type PVC gel artificial muscle using a meshed electrode as the anode [15]. The PVC gel is sandwiched between a stainless mesh as an anode and a stainless foil as a cathode. Fig. 3 shows the deformation mechanism of the PVC gel artificial muscles. When the DC field is applied, the PVC gel creeps up the anode and moves into the mesh hole, and the actuator shrinks in the direction of thickness. When the DC field is turned off, it returns to its former shape very quickly because of its elasticity. Thus it performs as an expansion and contraction deformation similar to a human muscle. And by stacking in layers we can get a multilayered contraction and expansion type artificial muscle (see Fig. 4).

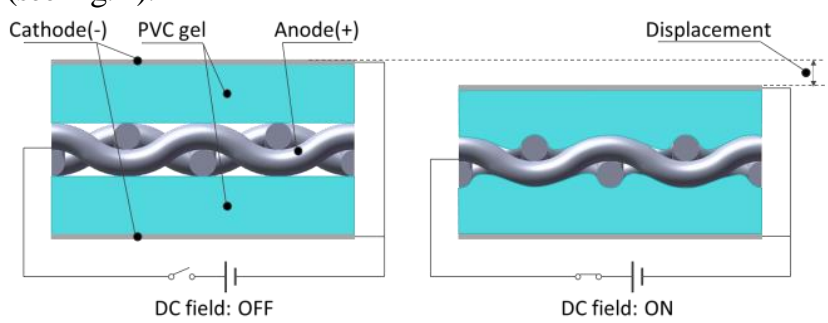

Fig. 3. Cross sectional deformation of a single layer PVC gel artificial muscle.

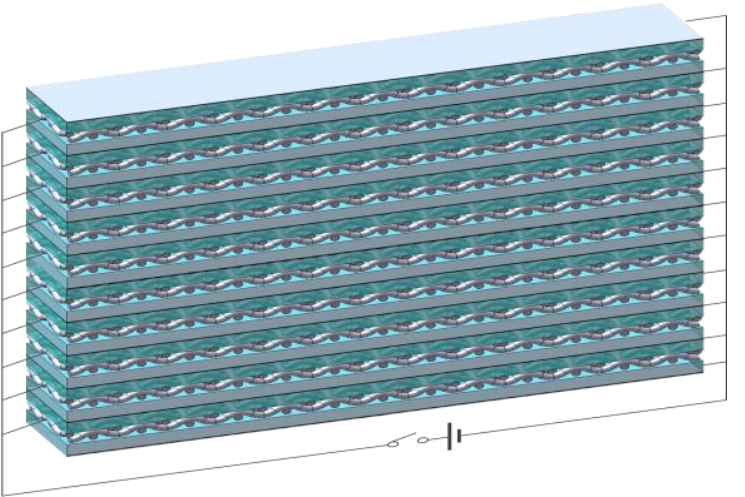

Fig. 4. Structure of 10-layer multilayered PVC gel artificial muscles.

\subsection{Characterizations}

Fig. 5 shows an example of fabricated 10-layer multilayered PVC gel artificial muscles, which is sandwiched by two glass slides at the top and bottom. The PVC gel artificial muscle has a dimension of about $50 \times 10 \times 6 \mathrm{~mm}$ and a weight of $5 \mathrm{~g}$. Table 1 shows the compositions and conditions of the samples in detail. 
We measured the displacement, recovery stress, electric current and response rate of the 10-layer PVC gel artificial muscle. The applied DC voltage is from $80 \mathrm{~V}$ to $400 \mathrm{~V}$.

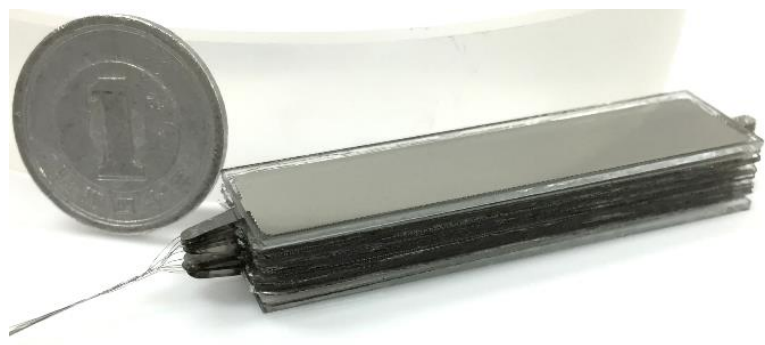

Fig. 5. A sample of fabricated 10-layer multilayered PVC gel artificial muscles with a One-yen coin.

\section{Table 1}

Compositions and conditions of fabricated PVC gel artificial muscles.

\begin{tabular}{|c|c|c|c|}
\hline & PVC gel & Anode & Cathode \\
\hline Material & PVC:DBA=1:4 & $\begin{array}{c}\text { Stainless } \\
\text { mesh (\#100) }\end{array}$ & Stainless foil \\
\hline Size & $50 \times 10 \times 0.2 \mathrm{~mm}$ & $\begin{array}{c}50 \times 10 \times 0.18 \\
\mathrm{~mm} \text { (wire } \\
\text { diameter of } \\
0.09 \mathrm{~mm} \text { ) }\end{array}$ & $\begin{array}{c}50 \times 10 \times 0.01 \\
\mathrm{~mm}\end{array}$ \\
\hline Number & 20 & 10 & 11 \\
\hline
\end{tabular}

\subsubsection{Displacement measurement}

The displacement in the thickness direction was measured by a laser displacement sensor (IL-065, KEYENCE, JAPAN). Fig. 6 shows the result of the displacement under variation of applied voltages. We can see the dependency of the displacement on the applied DC voltage. In the case of $400 \mathrm{~V}$, the displacement is about $0.72 \mathrm{~mm}$ and a contraction strain of $12 \%$, which is almost the same as that reported in the prior literature under an applied voltage of $900 \mathrm{~V}[16]$.

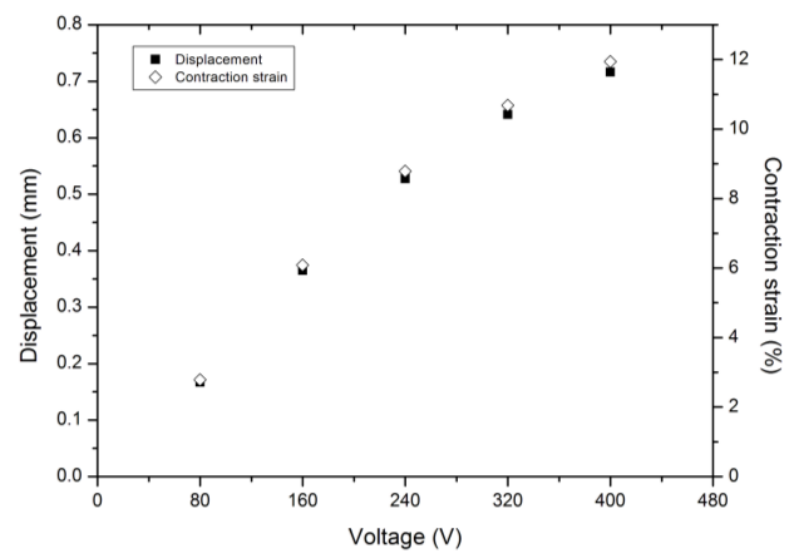

Fig. 6. Displacement and contraction strain of PVC gel artificial muscles.

\subsubsection{Recovery stress measurement}

When the DC field is applied, the electrical Maxwell force happens and leads to the contraction deformation of the PVC gel artificial muscle. When the DC field is turned off, the deformed PVC gel artificial muscle returns to its original form and generate a recovery force according to the elasticity of PVC gels. We measured this recovery stress as the generation force of this PVC gel artificial muscles. However, since the displacement of the artificial muscles is small, it is not ideal to use the strain gauge type load cell to measure the recovery force. There should be a big loss of force due to the strain of the load cell. In this study we use the strain-stress relation to measure the recovery stress. Fig.7 shows the method of the measurement. When the DC field is applied, we put a load on the artificial muscle. Then the load is uplifted by the upward pressure of artificial muscle when the DC is turned off. A laser displacement sensor is induced to measure the displacement of artificial muscle when the DC filed is turned off. With the applied load increases, the strain of artificial muscle decreases. By adjusting the load, we can get the relation between strain and stress. We define the stress at the strain of $0 \%$ as the maximum recovery stress of PVC gel artificial muscles. Fig. 8 shows the result of the recovery stress at different strains of each applied DC voltage. The recovery force also depends on the applied DC voltage. The maximum recovery force is about $78 \mathrm{kPa}(39 \mathrm{~N})$ when the applied voltage is $400 \mathrm{~V}$, which is almost 20 times as much as that reported in the prior literature under an applied voltage of 900V [16]. The PVC gel artificial muscles achieve a peak power-to-weight ratio of $3 \mathrm{~W} / \mathrm{kg}$, and a peak power-to-density ratio of $4.3 \mathrm{~kW} / \mathrm{m}^{3}$ at the applied voltage of $400 \mathrm{~V}$. The power-to-weight ratio is smaller than that of human biceps muscle of $50 \mathrm{~W} / \mathrm{kg}$, but reaches the same level of magneto elastic wave motor and exceed a piezoelectric motor of $0.1 \mathrm{~W} / \mathrm{kg}[24]$.
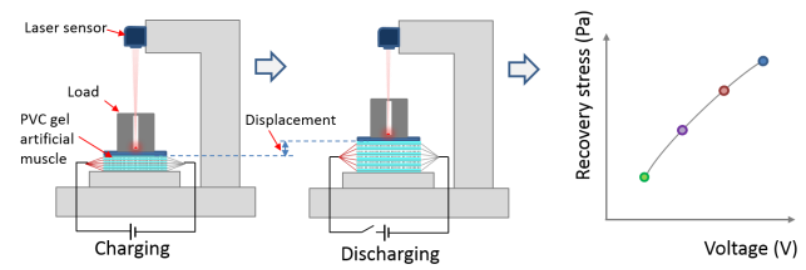

Fig. 7. Illustration of recovery stress measurement of PVC gel artificial muscles.

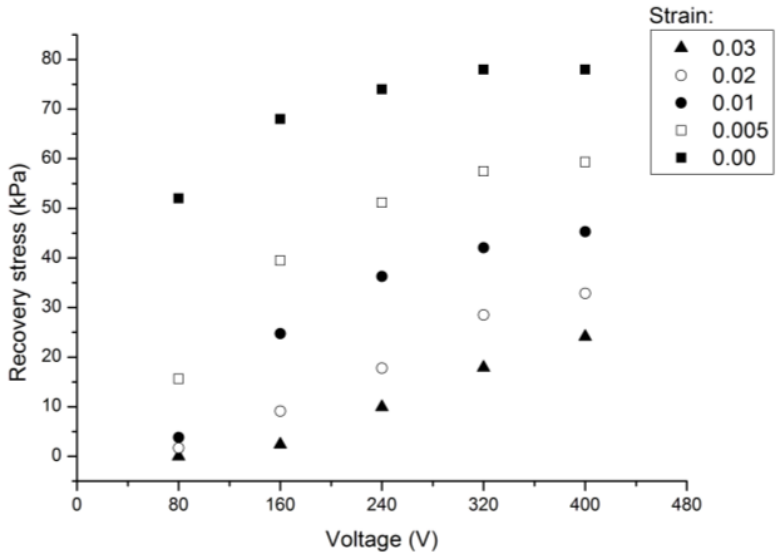

Fig. 8. Recovery stress of PVC gel artificial muscles.

\subsubsection{Electrical current measurement}

As one of the evaluation criteria of artificial muscles, the power consumption becomes an important parameter. We introduced a series resistor of $1 \mathrm{k} \Omega$ to evaluate the electrical current of PVC gel artificial muscles. As shown in Fig. 9 that an instantaneous inrush current occurs when the DC field is turned on and decrease dramatically to a stable current, and a negative inrush current occurs when the DC field is turned off and decrease to zero quickly. This is due to the charge and discharge of the capacitance of PVC gel which is consistent with the result 
of the equivalent circuit of PVC gel artificial muscles in prior literature [18]. Fig. 10 shows the variation of the stable current of PVC gel actuators. The electrical current increases with the applied DC fields. The current of the 10-layer artificial muscle is about $0.036 \mathrm{~mA}(14.4 \mathrm{~mW})$ under an applied DC voltage of $400 \mathrm{~V}$ which indicates that the PVC gel artificial muscles have a low power consumption.

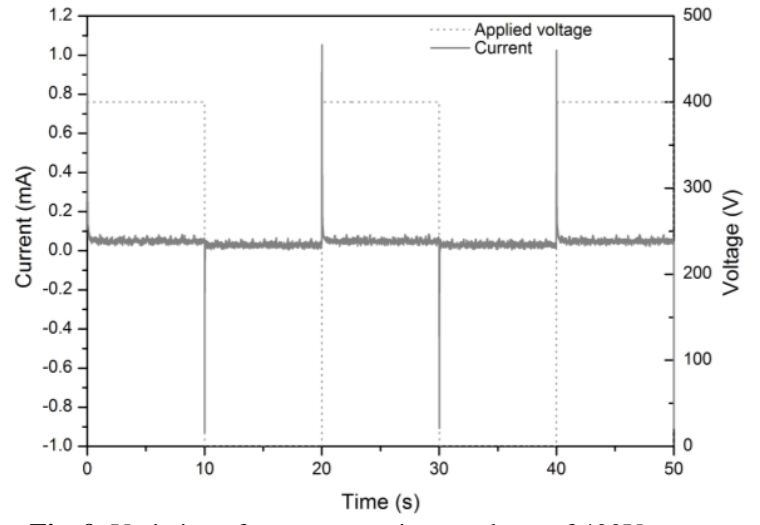

Fig. 9. Variation of current at an input voltage of 400V.

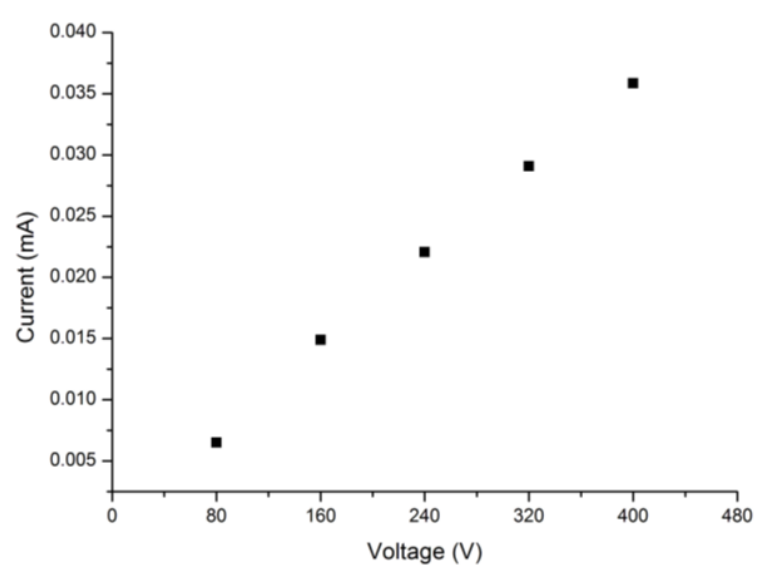

Fig. 10. Current of PVC gel artificial muscles.

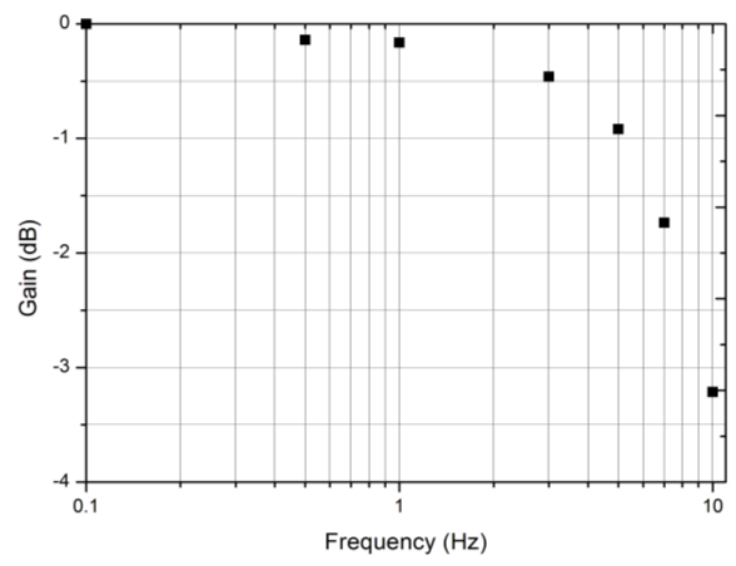

Fig. 11. Response rate of PVC gel artificial muscles.

\subsubsection{Response rate measurement}

The response rate was investigated by the frequency response method. The input signal to the artificial muscles was rectangular pulses with the frequencies from $0.1 \mathrm{~Hz}$ to $10 \mathrm{~Hz}$. The applied DC voltage of the rectangular pulse was $400 \mathrm{~V}$. Fig. 11 shows the bode diagram of the displacement. The band width $(-3 \mathrm{~dB})$ of the PVC gel artificial muscles is $9 \mathrm{~Hz}$ which reaches the same level of biological muscles [25].

\subsubsection{Cycle life evaluation of PVC gel artificial muscles}

In recently decades, a variety of artificial muscles that exhibit substantial deformations in response to different external stimulus have been developed. However, a very few of them have demonstrated exceptional cycle life, which should be one of the reasons that no single artificial muscle technology that has received wide-spread adoption as an artificial skeletal muscle so far. Among the few artificial muscles, a swaged pneumatic artificial muscle [26] and a fishing line based artificial muscle [27] exhibit a cycle life over 1 million actuation cycles.

Here, we evaluated the durability of the PVC gel artificial muscle under a continuous electric field for a long period to ensure that its cycle life is sufficient for practical applications. An applied voltage of $320 \mathrm{~V}$ (a sine wave of $\pm 160 \mathrm{~V}$, of which the origin was shifted from zero to $160 \mathrm{~V}$ ) with a frequency of 2 $\mathrm{Hz}$ was continuously applied to the PVC gel artificial muscles for about 31 days (about 5.4 million cycles). Fig. 12 shows the method of the durability evaluation experiment. Three single-layer PVC gel actuators were evaluated at a constant room temperature of $20^{\circ} \mathrm{C}$ in a thermostatic chamber.

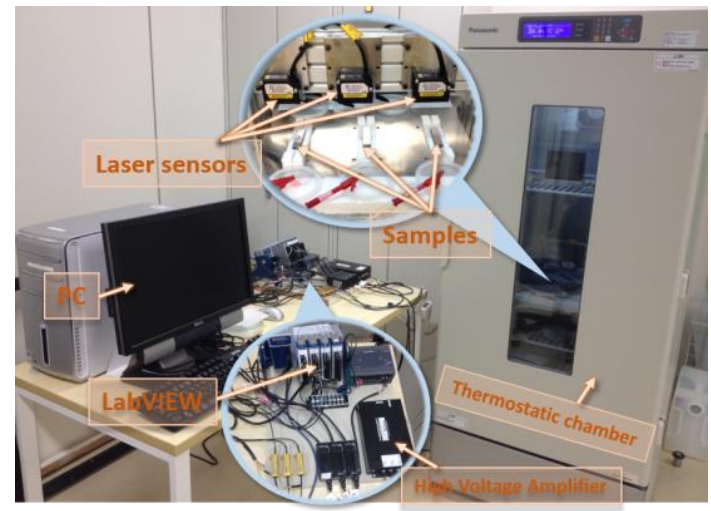

Fig. 12. Photograph of the measurement system.

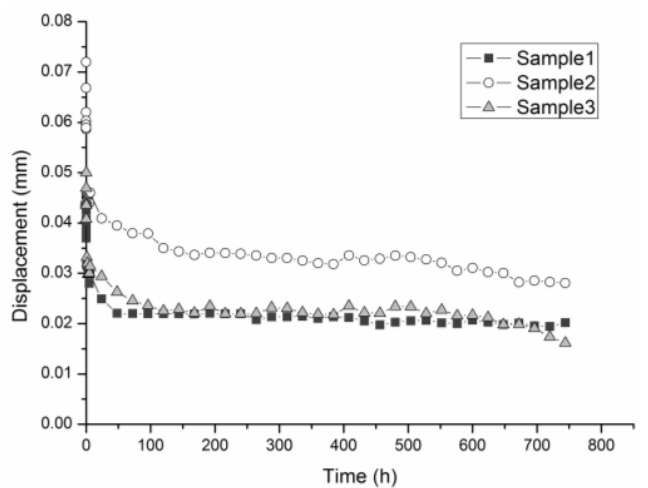

Fig. 13. Variation of displacement during a long-term experiment.

Fig. 13 shows the result of displacement variation during the durability experiment. The displacements of the three samples decreased to about $64 \%$ of the maximum displacement after 6 hours, $50 \%$ after 168 hours (7 days) and remained constant until 600 hours (25 days). However, no notable change of the recovery stress was confirmed before and after the durability experiment. Furthermore, we found an increasing trend of displacement after a break time, which can be attributed to the recovering shape of PVC gel from a long-term deformation due to the complex structure of the stainless mesh anode. Therefore it was confirmed that the PVC gel artificial muscles' cycle life is more than 5 million times at the state of continuous electric field 
driven $(2 \mathrm{~Hz})$, which reaches a level closes to a mammalian skeletal muscle [28]. It shows a great advantage over many other artificial muscles, such as 3000 actuation cycles of IPMC [29].

As all the properties mentioned above, the PVC gel artificial muscles are light in weight, having good performances of large displacement, high output force, low power consumption and long cycle life, and therefore suitable for various of practical applications.

\section{Proposal of modular constructions of PVC gel artificial muscles}

Although the PVC gel artificial muscles are capable of generating large strain and strain rate. However, the multilayered PVC gel artificial muscles are just simply stacked by the unit structures of PVC gels, mesh anodes and foil cathodes. And each of the artificial muscle units moves independently under the electric fields. As long as a micro nonuniform structure of mesh anodes or PVC gels happens, the multilayered artificial muscles separate from one layer to the others when being pulled by a certain force under the electric fields. Consequently, the contraction force generated under the electric fields cannot be efficiently utilized in the practical applications. Though Ogawa et al [30] tried to solve this problem by enlarging the mesh anode of each layer and connecting them together to give a prepressing to enhance the contact between the gels and mesh anodes, it brought a big loss of strain and got an unsatisfactory contraction stress of $1.9 \mathrm{kPa}$. And we applied a thin coating of PVC gel on the surface of multilayered PVC artificial muscles to prevent the breaking, and tried to use the variable stiffness generated by the contraction force to develop PVC gel spats for walking assistance [20]. However, we only got a maximum tensile strain of $7 \%$, with a generation force of $2.63 \mathrm{~N}(5.26 \mathrm{kPa})$ until a break happened. The output stress is under one quarter of the recovery stress that we mentioned in section 3.2.2.

In this study, considering the drawback that mentioned above, we propose to use the recovery stress of PVC gel artificial muscles for the practical applications. Meanwhile, we propose three types of actuation modular constructions for different purposes of applications, so as to make PVC gel artificial muscles being robust actuation devices.

\subsection{Compression type actuation module structure}

The proposed compression type actuation module, as shown in Fig.14, being defined as that the actuation module can generate a compressive force without a break under an external compressive force. The multilayered PVC gel artificial muscle is enclosed in a case, with the top layer connected to a force transferring unit. To avoid the separation between the artificial muscle and the transferring unit, we fixed two mesh anodes on the bottom sides of the transferring unit and the container, respectively.

When the DC field is on (left part of Fig.14 (1), (2)), the artificial muscle shrinks in the direction of thickness. When the DC field is off, the artificial muscle recovers to the original shape and generate a force. The stroke and output force of the module can be controlled by the input voltage. The shape, size and other detailed design specifications of the case and artificial muscles can be adjusted according to the practical applications. Furthermore, we can get more displacement by the series connection of the actuation module units and bigger output force by the parallel connection of the actuation module units. In this case, the connection between each unit should be a rigid connection. Meanwhile, even though this type of actuation module units is not robust to the stretching force, we can make a more robust compressive and stretching actuation device by connecting the connector parts of the compression type actuation module units in I-shaped.

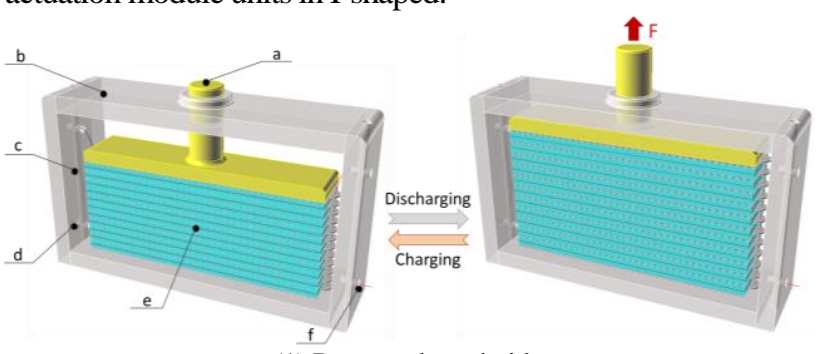

(1) Rectangular cuboid type

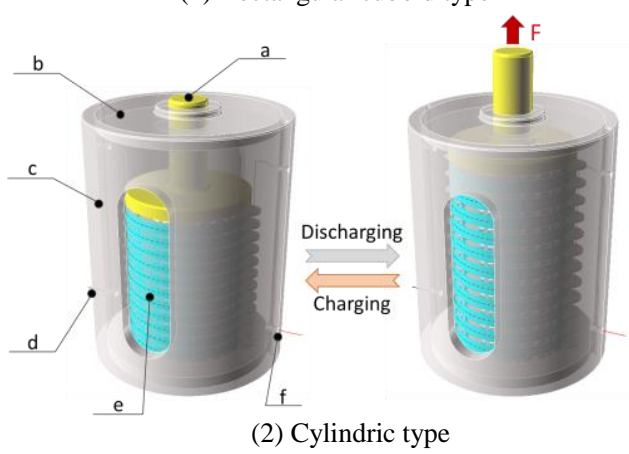

a: connector and force transferring part;

b: container and protector;

c: pipeline for wiring and aeration (left and right);

d: input of cathode (-);

e: PVC gel artificial muscle;

f: input of anode (+).

Fig. 14. Illustration of compression type actuation modules.

\subsection{Stretching type actuation module structure}

When the applications need a pulling force, for example, walking assist application, it is effective to design the actuation module as the structure shown in Fig.15. It mainly contains a slide frame and a container. The multilayered PVC gel artificial muscle is placed on the slide frame, of which the height is adjusted to the thickness of artificial muscles so as to prevent a hardly break when bearing a compressive load. And we can adjust the part of the connector and force transferring ('a' of Fig.15 (1), (2)) to control the preload to the artificial muscle. To avoid the separation among the layers of artificial muscles due to the self-weight of the slide frame, we fixed two mesh anodes on the top side and the bottom side of the container and the slide frame, respectively.

When the DC field is applied to the artificial muscle, it contracts and brings the slide frame an upward movement. And when the DC field is turned off, the artificial muscle occurs a downward movement and generate a tensile force without a break happens. Also, it is reasonable to connect the stretching actuation modules in a series connection or a parallel connection to adopt the practical applications. In this case, flexible connection is suitable for the connection among the modules, for example, using stainless wire. Although this type of actuation module units is not robust to the external compressive force, we can make a more robust compressive and stretching actuation device by connecting the connector parts of the 
stretching type actuation module units in I-shaped to make a device that is robust in both upward and downward movements.

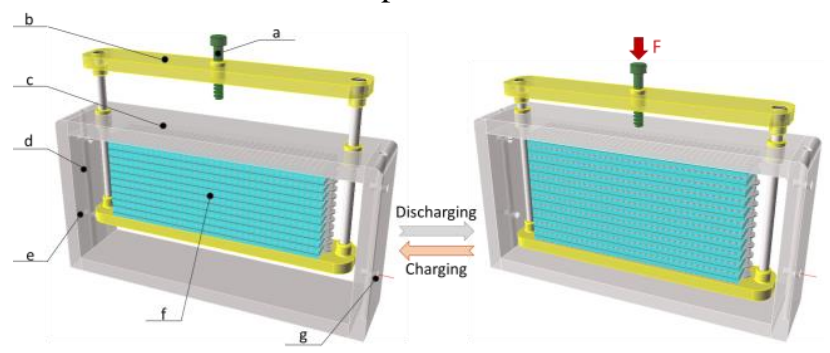

(1) Rectangular cuboid type

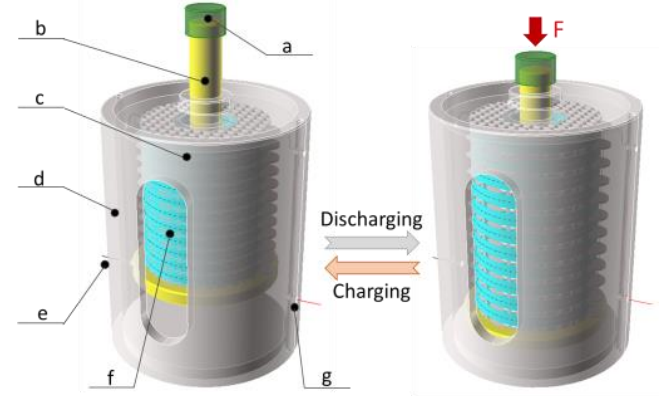

(2) Cylindric type

a: connector and force transferring part;

b: slide frame;

c: container and protector;

$\mathrm{d}$ : pipeline for wiring and aeration (left and right)

e: input of cathode (-);

f: PVC gel artificial muscle;

$\mathrm{g}$ : input of anode $(+)$.

Fig. 15. Illustration of stretching type actuation modules.

\subsection{Compression-Stretching type actuation module structure}

By combination of the compression type and stretching type actuation modules, we can make a compression and stretching type actuation module as shown in Fig.16. The basic structure is the same as the stretching type that contains a slide frame and a container. Two multilayered PVC gel artificial muscles are arranged inside and outside of the slide frame. Both the top and bottom sides of the upper artificial muscle are fixed to the container and slide frame, respectively. And the top and bottom sides of the lower artificial muscle are fixed to the bottom side of slide frame and the container, respectively.

When the upper artificial muscle is charged and the lower artificial muscle is discharged at the same time, the actuation module produces a compressive force. Otherwise, when the upper artificial muscle is discharged and the lower artificial muscle is charged coinstantaneous, a pulling force occurs. The interaction force between the upper and lower artificial muscles can prevent the break in both the contraction and expansion movement of the slide frame. Therefore, this actuation module type is robust in the bidirectional assistance.

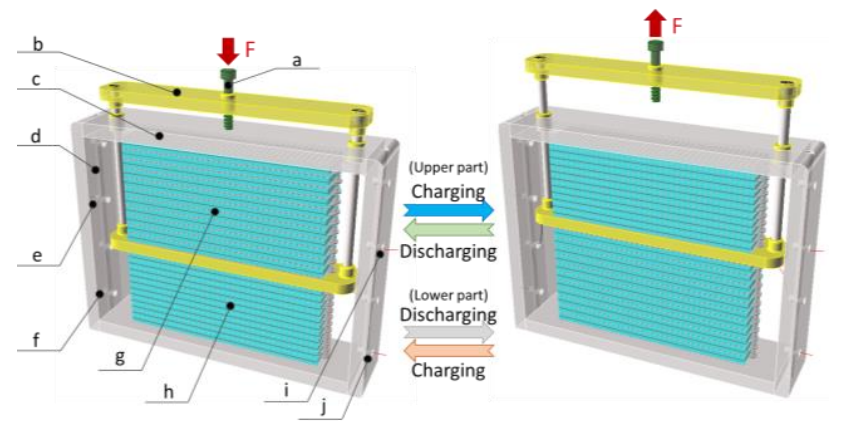

(1) Rectangular cuboid type

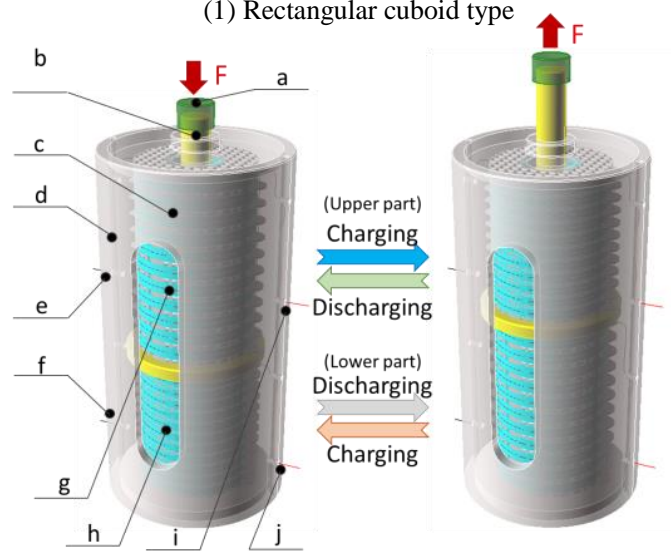

(2) Cylindric type

a: connector and force transferring part;

b: slide frame;

c: container and protector;

$\mathrm{d}$ : pipeline for wiring and aeration (left and right);

e: input of cathode (-) of the upper artificial muscle;

f: input of cathode (-) of the lower artificial muscle;

g: upper PVC gel artificial muscle;

h: lower PVC gel artificial muscle;

$\mathrm{i}$ : input of anode $(+)$ of the upper artificial muscle.

$\mathrm{j}$ : input of anode $(+)$ of the lower artificial muscle.

Fig. 16. Illustration of compression-stretching type actuation modules.

\section{Experimental}

In this study, we made a prototype of stretching type actuation module to evaluate the effectiveness of the proposed modules. We investigated the advantages of using recovery force of PVC gel artificial muscles instead of contraction force using the fabricated stretching type actuation module, from two aspects of robustness and response time against the external loads. Furthermore, as a design and control element of the proposed modules in practical use, we developed a model of static characteristics of PVC gel artificial muscles based on the experimental data measured by the prototype of modules.

\subsection{Prototype of module units}

Fig. 17 shows the overview of the fabricated stretching type module unit. It mainly contains of a slide frame that made of Aluminum plate and a container that made of Aluminum and Teflon plates. In order to decrease the frication force of sliding movement, we introduced a linear bearing (LMUF3, MISUMI, JAPAN) to the slide frame. And the length of the slide frame was adjustable so as to fit the thickness of artificial muscles.

A 10-layer multilayered PVC gel artificial muscle was fixed between the bottom of slide frame and the top of the container. The compositions and conditions of the artificial muscle was the same as shown in Tab.1 in section 3.2. When making the 
actuation movement without an external load, to avoid the separation among the layers of artificial muscles due to the self-weight of the slide frame, we fixed two mesh anodes on the top side and the bottom side of the container and the slide frame, respectively.

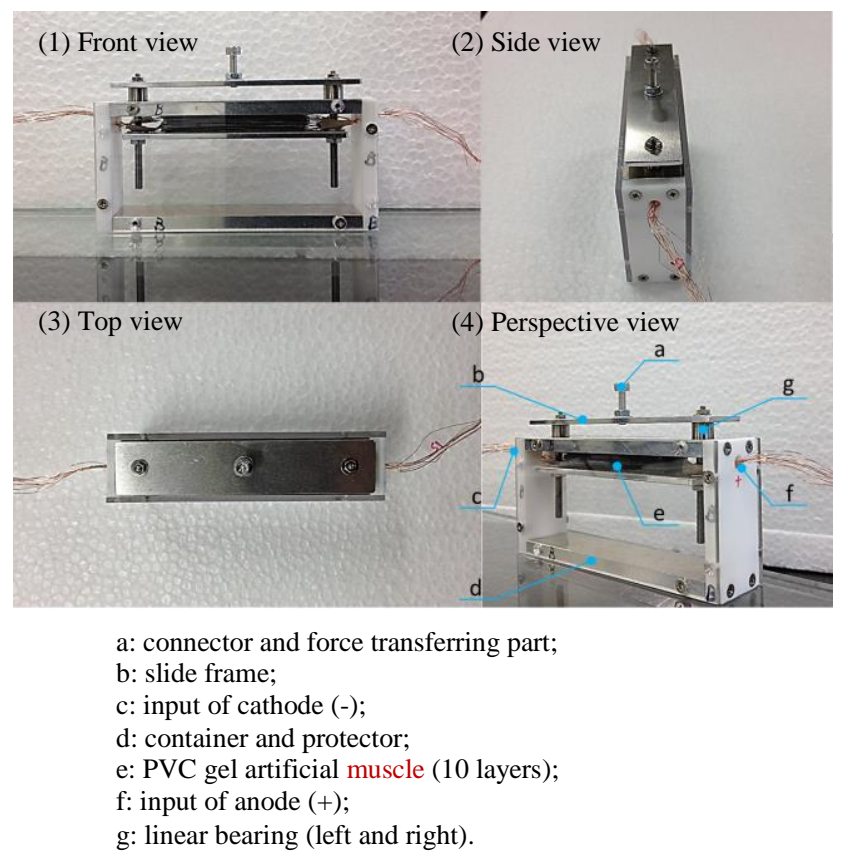

Fig. 17. A prototype of stretching type actuation modules.

\subsection{Performance evaluation experiment}

\subsubsection{Methods and conditions}

In this study, based on the prototype of stretching type actuation module, we conducted a comparison experiment between the contraction force and recovery force to confirm the performances and advantages of the proposed actuation modules that using the recovery force of PVC gel artificial muscles. Fig.18 shows the method of the evaluation experiment. As shown in Fig.18 (a), to evaluate the performance of contraction force of the actuation module, we put the loads on the top of the slide frame of actuation module. When the DC field is applied to the artificial muscle, it shrinks and a contraction force $\boldsymbol{F}_{\boldsymbol{c}}$ generated to lift the load upward. And it returns to its original shape due to the elasticity of PVC gel and the applied load when the DC field is turned off. A laser sensor is used to measure the displacement of the load. As we mentioned before that each of the artificial muscle units moves independently under the electric fields. With the increase of the applied loads, the displacement of the load decreases and the multilayered artificial muscle separates from one layer to the others when being compressed by a certain force due to the nonuniform structure of mesh anodes or PVC gels. As a result, the contraction force generated before the break can be efficiently utilized in the practical applications.

On the other hand, for the recovery force evaluation of the actuation module, we designed a measurement device as shown in Fig.18 (b). The actuation module is connected to the applied load by stainless wire and pulleys. When a DC field is applied to the artificial muscle, it shrinks and brings a downward movement to the external load. And when the DC field is turned off, the artificial muscle recovers to its original shape by its elasticity very quick, generating a recovery force $\boldsymbol{F}_{\boldsymbol{r}}$ and consequently lift the applied load upward. The displacement of the applied load is measured by displacement of the slide frame. By this utilization of the stretching type actuation module, there will not be a break happens among the layers of PVC gel artificial muscles. With the applied load increases, the strain of artificial muscle decreases and the stress at the strain of $0 \%$ is the maximum recovery stress of PVC gel artificial muscles that we can efficiently utilize in the practical applications.

In this experiment, we compared the variation of both the contraction force and recovery force under a DC voltage of $400 \mathrm{~V}$. Also the response time of the two kinds of forces was investigated. As shown in Fig.19, the response time of contraction force $\left(\boldsymbol{t}_{\boldsymbol{c}}\right)$ and recovery force $\left(\boldsymbol{t}_{\boldsymbol{r}}\right)$ is defined as the time from $10 \%$ to $90 \%$ of the contraction displacement and recovery displacement, respectively.

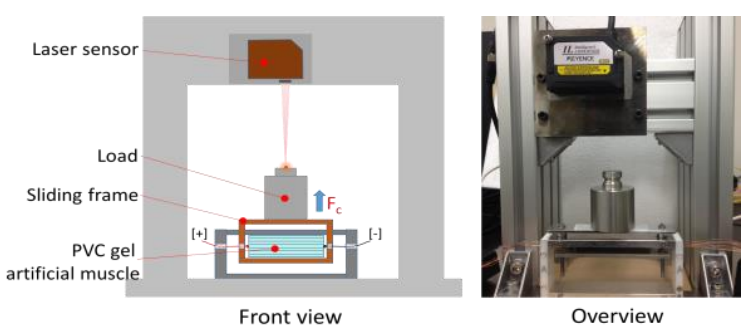

(a) Performance measurement of contraction force

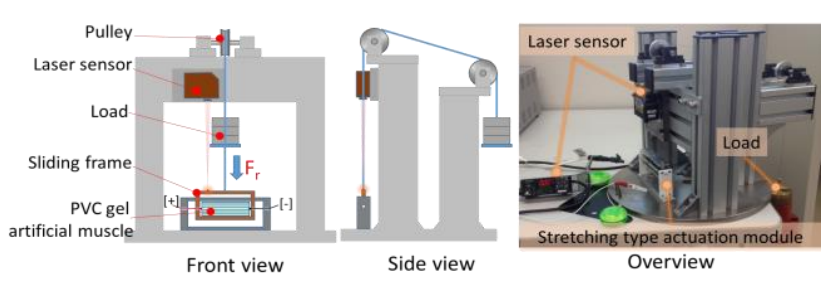

(b) Performance measurement of recovery force

Fig. 18. Method of evaluation experiment.

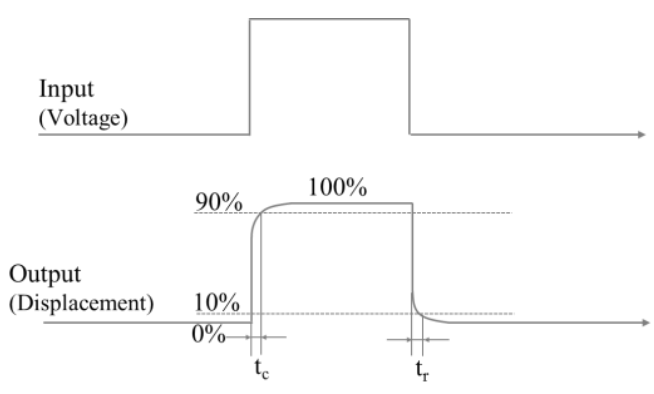

Fig. 19. Definition of response time of contraction and expansion.

\subsubsection{Results of experiment}

Fig.20 shows the result of the comparison of contraction force and recovery force measurement. As shown in the result of contraction force and strain, the strain drops dramatically to $0 \%$ at the applied load of $3.5 \mathrm{~N}(7 \mathrm{kPa})$ which shows that the maximum generation force that can be used before a break happens is below $3.5 \mathrm{~N}$. However, as for the recovery force and strain, we can see that the maximum generation force is about $38 \mathrm{~N}(78 \mathrm{kPa})$ at the strain of $0 \%$ which is about 11 times of the maximum contraction force.

Fig.21 shows the result of the response time of contraction force and recovery force. The response time $t_{r}$ of recovery force is about $57 \mathrm{~ms}$, which is slow than that of contraction force $\boldsymbol{t}_{c}$ 
(about 36ms). But we don't consider it as a big difference in the practical applications. Since the response time of the recovery force is determined by the viscoelasticity of PVC gel, we can adjust the weight ratio of PVC and DBA to improve the response speed.

Therefore, as discussed above, it is obvious that the proposed module constructions are effective to utilize the properties of PVC gel artificial muscles so as to make the PVC gel artificial muscles as a kind of robust actuation devices in various applications from some micro machines to large scale devices.

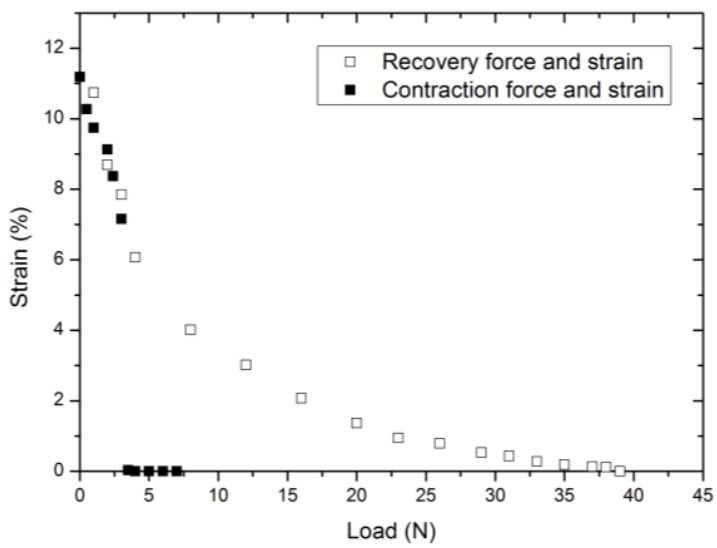

Fig. 20. Result of contraction force and recovery force measurement.

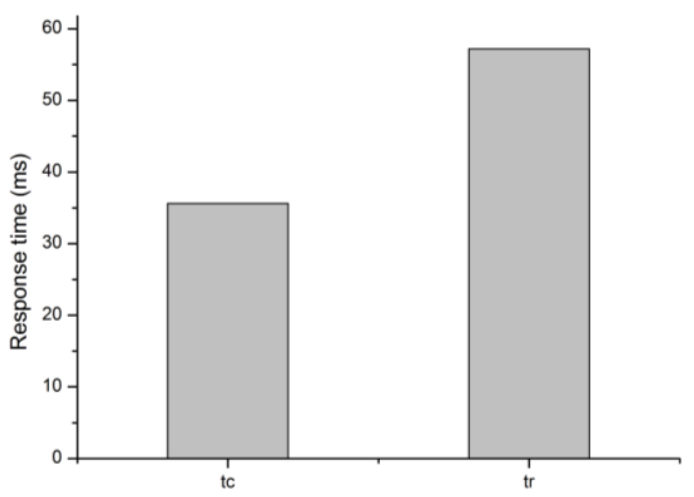

Fig. 21. Result of response time of contraction force and recovery force.

\subsection{Module unit based characteristics evaluation and modeling}

\subsubsection{Characteristics with different number of stacked layers}

In this study, based on the prototype of stretching type actuation module (Fig.17), we conducted a characteristics evaluation experiment of PVC gel artificial muscles with different number of stacked layers. The method of measurement was the same as shown in Fig.18 (b).

Several 5-layer and 10-layer PVC gel artificial muscles, with the same conditions of material and size as shown in Table1, were used in this experiment. We measured the relation between displacement and output force with the applied DC voltages from $80 \mathrm{~V}$ to $400 \mathrm{~V}$.

Fig.22 shows the results of the relation between displacement and output force of PVC gel artificial muscles under different applied voltages. An inversely proportional relationship (as part of a rectangular hyperbola) was confirmed between displacement and output force. At the same applied voltage, with the increase of the displacement, the output force decreases. And for the same displacement (or output force), with the increase of number of stacked layers, the output force (or displacement) increases. Besides, with the increase of the applied voltage, the maximum displacement and output force increase.
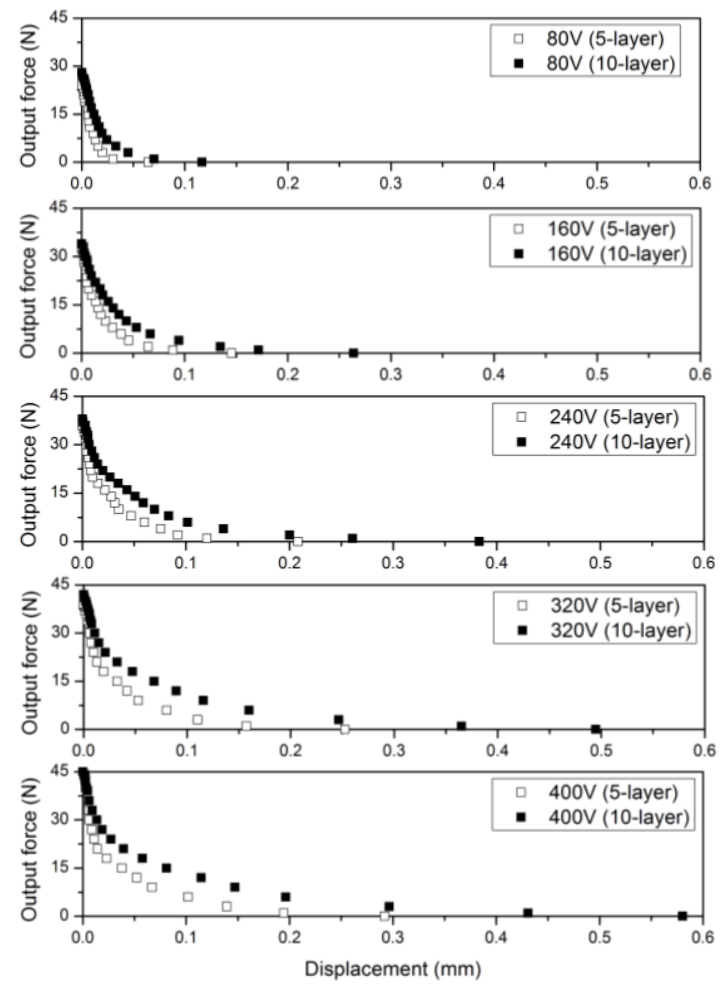

Fig. 22. Relation between displacement and output force under different applied DC voltages.
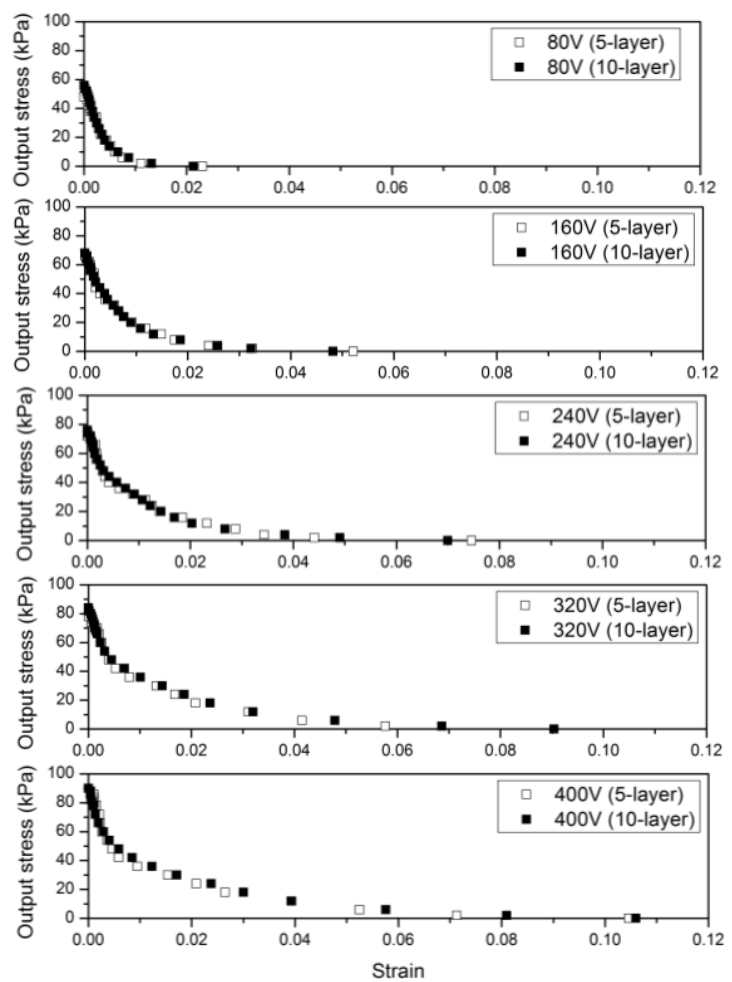

Fig. 23. Relation between strain and output stress under different applied DC voltages.

Fig.23 shows the results of relation between strain and output stress of PVC gel artificial muscles under different applied DC 
voltages. As shown in the results, for all the applied voltages, the curves of the 5-layer artificial muscles are almost the same as that for the 10-layer artificial muscles. This indicates that, under a certain applied DC voltage, the displacement of PVC gel artificial muscles is linear proportional to the number of stacked layers. And the output stress remains constant with the variation of the number of stacked layers.

\subsubsection{Modeling of characteristics}

Considering practical applications of the proposed actuation modular constructions, as a design and control element, we modeled the static characteristics of PVC gel artificial muscles based on the experimental results that measured by the prototype of stretching type actuation module.

In this study, we developed a model to represent the relation between the applied DC voltages and output of displacement and force based on Hill's muscle model [31]. Hill demonstrated that the force-velocity relation in skeletal muscle can be described as part of a rectangular hyperbola as shown in Fig.24. And the general equation of this relation given as the Hill muscle model as follows:

$$
\begin{aligned}
& (P+a)(S+b)=\left(P_{m}+a\right) b=C, \\
& \frac{P}{P_{m}}=\frac{S}{S_{m}}=\text { constan } t
\end{aligned}
$$

where $P$ and $S$ are the muscle force and the contraction velocity, respectively. $a, b$ and $C$ are constants. $P_{m}$ is the maximum force with zero velocity, and $S_{m}$ is the maximum contraction velocity with zero force.

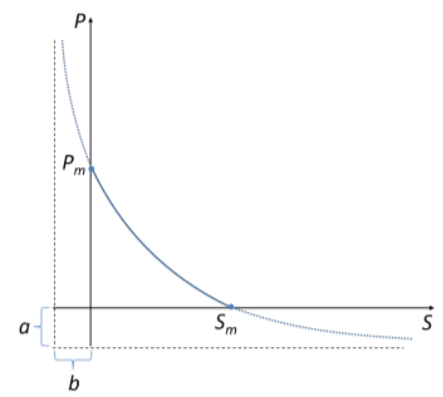

Fig. 24. Hill's force-velocity relationship, where $P_{m}$ is maximum isometric force or force with zero velocity, $S_{m}$ is the highest possible velocity, $a$ and $b$ are constant force and constant velocity.

Since the curve of displacement and output force of PVC gel artificial muscles is quite similar with Hill's muscle model, we modeled the relation between displacement and output force of PVC gel artificial muscles as described in the following equation:

$$
\left(F+a_{f}\right)\left(X+b_{x}\right)=\left(F_{m}+a_{f}\right) b_{x}=\left(X_{m}+b_{x}\right) a_{f}=c
$$

where $F$ and $X$ are the output force and the displacement, respectively. $a_{f}, b_{x}$ and $c$ are constants. $F_{m}$ is the maximum force with zero displacement, and $X_{m}$ is the maximum displacement with zero force.

$F$ and $X$ can be obtained by the experimental data. $a_{f}, b_{x}$ and $c$ can be obtained by fitting Hill's hyperbolic equation to experimental data using regression analysis of the variables $F, X$ and $F X$ in the hyperbolic equation [32]. We used the experimental data of 10-layer PVC artificial muscles to calculate the numerical values of the three constants. The obtained $a_{f}, b_{x}$ and $c$ with different applied DC voltages are shown in Fig. 25.

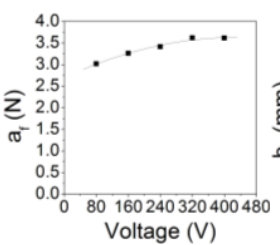

(a)

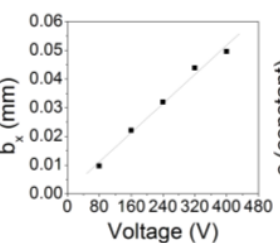

(b)

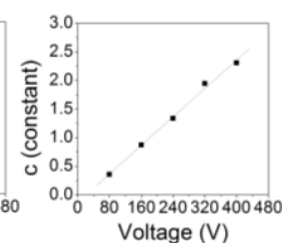

(c)
Fig. 25. Results of constant coefficients at different voltages. (a) $a_{\mathrm{f}}$. (b) $b_{\mathrm{x}}$. (c) c.

The relation between the constants and applied DC voltages can be presented as follows:

$$
\begin{aligned}
& A_{f}(v)=-5.0176 \times 10^{-6} v^{2}+4.3400 \times 10^{-3} v+2.6956 \\
& B_{x}(v)=1.2700 \times 10^{-4} v+9.7018 \\
& C(v)=6.2100 \times 10^{-3} v-1.4925
\end{aligned}
$$

Therefore, we can model the relation between the applied DC voltages, displacement and output force of PVC gel artificial muscles by Eq. (7):

$$
\left(F+A_{f}(v)\right)\left(X+B_{x}(v)\right)=C(v)
$$

Fig.26 shows the simulation result of the model and the measured data of 10-layer artificial muscles. This indicates a good agreement between the model and the measured data. For a practical application, once two of the desired parameters of applied DC voltage, displacement and output force in the model are defined, we can obtain the rest one. Since we have addressed in Fig.23 that the displacement of PVC gel artificial muscles is linear proportional to the number of stacked layers and the output stress remains constant with the variation of the number of stacked layers, this model can be also effective to different number of stacked layers by simply normalize the displacement with the number of stacked layers.

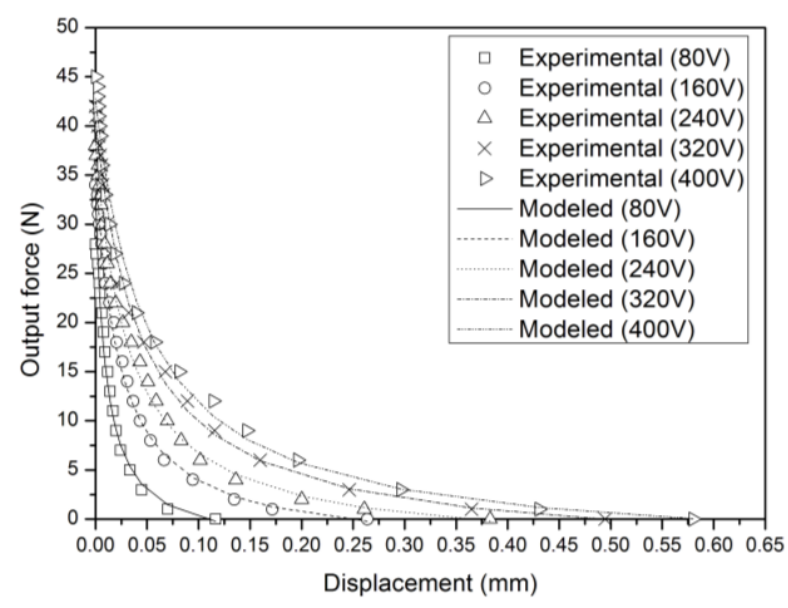

Fig. 26. Simulation result based on the proposed model.

When the requirement of an application is determined, we have to design the actuation module to satisfy it. Then we have to determine the structure and size of the actuation module according to the size of artificial muscles. PVC gel artificial muscles' size is determined by the number of stacked layers and the area of each layer. And the displacement is linear proportional to the number of stacked layers and the output force is linear proportional to the area of each layer. To find the relation between the number of stacked layers and the output stress, we can define the $F$ and $X$ of Eq. (7) as follows: 


$$
X=\frac{10 X_{\text {desire }}}{N_{\text {layer }}}
$$

$F=A_{\text {area }} S_{\text {stress }}=5 \times 10^{-4} S_{\text {stress }}$

where $X_{\text {desire }}$ is the displacement required by an application. $N_{\text {layer }}$ is the number of stacked layers. $A_{\text {area }}$ and $S_{\text {stress }}$ are the area of one layer and the output stress, respectively. Then, the model of relation between number of stacked layers and output stress can be given as Eq. (10).

$\left(5 \times 10^{-4} S_{\text {stress }}+A_{f}(v)\right)\left(\frac{10 X_{\text {desire }}}{N_{\text {layer }}}+B_{x}(v)\right)=C(v)$

Fig.27 shows the simulation result of a desired displacement of $0.5 \mathrm{~mm}$. At the same number of stacked layers, with the increase of applied DC voltage, the output stress increases. And at the same applied DC voltage, with the increase of number of stacked layers, the output stress increases. The output force is determined by the area and stress and the height of artificial muscles is determined by the number of stacked layers. Therefore, using this model we can get an optimization design of the size of the actuation modular constructions for a practical application, for example, an application as a motor brake.

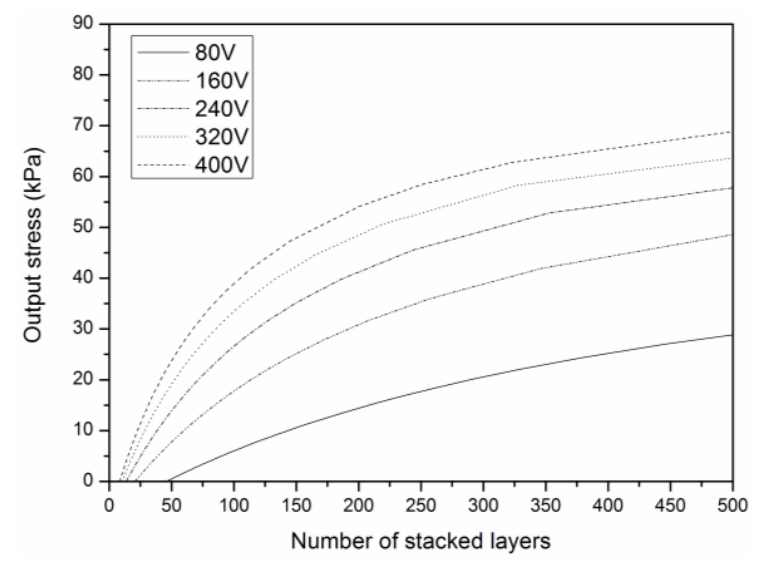

Fig. 27. Relation between the number of stacked layers and the output stress at a desired displacement of $0.5 \mathrm{~mm}$.

\section{Discussions}

Although a variety of artificial muscles have so far been developed and exhibited different advantages compared to the conventional mechanical actuators, most of them are being challenged by the characteristics improvement to reach or exceed the level the nature muscles for a successful commercialization and a wide use. That is to achieve a large strain and stress, a fast response rate and a long cycle life with a low power consumption. Meanwhile, with the progress of the characteristics improvement, it is also important to take the modeling, controlling, standardization and fabrication into consideration.

PVC gel artificial muscles have been studied for several years in our research and we have gotten properties that almost close to the level of the nature muscles, especially in terms of stable contraction strain and response rate in the air. And the maximum stress is about one quarter of the maximum stress of nature muscles. These advantages along with the satisfactory cycle life indicate that the PVC gel artificial muscles have a great potential to use as a candidate of artificial muscles in practical use. The three types of modular constructions proposed in this study are very important elements for the standardization of PVC gel artificial muscles. They make the PVC gel artificial muscles become robust actuation devices. By combining them in different constructions with different functions we can realize various applications. For example, we can make a robust and lightweight walking assist wear by combining the stretching type actuation module units in a series connection. We can support the walking motion by the output force generated with the DC field on and off, so as to lighten the load of human muscles. Furthermore, the model of the characteristics developed in this study which showed a good agreement with the experimental data could be an effective element for the specific design and control of the proposed modular constructions in an application. In future, we will do further research to improve the characteristics and decrease the input voltage for the safety. And we believe that the PVC gel artificial muscles based actuation modular units will play an important role in the field of mechatronics, robotics, biomimetics and biomedicine in the future.

\section{Conclusions}

In this study, we introduced the most updated characteristics of PVC gel artificial muscles to show the potential as a candidate of artificial muscles in practical use. In addition, as one important step for the practical application, we proposed three types of actuation modular constructions to make the PVC gel artificial muscles as a robust actuation device.

In view of the drawback of utilizing the contraction force, we proposed to use the recovery stress of PVC gel artificial muscles for the practical applications. Based on this view, we proposed a compression type, a stretching type and a compression-stretching type actuation modular constructions for different purposes of applications. And a prototype of stretching type actuation module unit was fabricated to confirm the effectiveness of the proposed modules. The results showed the reasonability of the proposed modular constructions. And we developed a model of the characteristics of PVC gel artificial muscles based on the experimental data measured by the prototype of module units, which could be effective in designing and controlling of the actuation modular constructions.

PVC gel artificial muscles based actuation modules are capable of generating a stable movement in the air without any noise, with a large strain and stress, a fast response rate with a low power comsumption. In addition to these advantages, a more than 5 millon times' cycle life is also sufficient for practical uses. Therefore, as an alternative among the artificial muscles or the traditional mechanical actuators, many applications are expected.

\section{Acknowledgment}

This work was supported by Shinshu Medical Industry Association of Japan and by JSPS KAKENHI Grant Number $15 \mathrm{~K} 18002$.

\section{References}

[1] T. Mirfakhraia, J. D.W. Maddena, R. H. Baughman, Polymer artificial muscles, Mater. Today 10 (2007) 30-38.

[2] K. Asaka, H. Okuzaki, Soft actuators, materials, modeling, applications and future perspectives, Polymer science, 2014.

[3] S. Sherrit, X. Bao and Y. Bar-Cohen, Methods of testing and characterization, in: Bar-Cohen, Y., (Ed.), Electroactive Polymer (EAP) Actuators as Artificial Muscles, 2nd Edition, SPIE Press, Vol. PM136, 2004, pp. 467-526.

[4] Y. Nabetani, H. Takamura, Y. Hayasaka, T. Shimada, S. Takagi, H. Tachibana, D. Masui, Zhiwei T. and H. Inoue, A photoactivated 
artificial muscle model unit: reversible, photoinduced sliding of nanosheets, J. Am. Chem. Soc. 133 (2011) 17130-17133.

[5] F. Daerden, D. Lefeber, B. Verrelst and R. Van Ham, Pleated pneumatic artificial muscles: actuators for automation and robotics, in: Proceedings of 2001 IEEE/ASME International Conference on Advanced Intelligent Mechatronics, 2001, pp.738-743.

[6] F. Daerden, D. Lefeber, Pneumatic artificial muscles: actuators for robotics and automation, Eur. J. Mech. Environ. Eng. 47 (2002) 11-21.

[7] J. Leng, X. Lan, Y. Liu, S. Dua. Shape-memory polymers and their composites: Stimulus methods and applications. Prog. Mater. Sci. 56 (2011) 1077-1135.

[8] Y. Liu, H. Du, L. Liu and J. Leng, Shape memory polymers and their composites in aerospace applications: a review, Smart Mater. Struct. 23 (2014) 1-22.

[9] S. Nemat-Nasser and Y. Wu, Comparative experimental study of ionic polymer-metal composites with different backbone ionomers and in various cation forms, J. Appl. Phys. 93 (2003) 5255-5267.

[10] S. G. Lee, H. C. Park, Surya D. Pandita, and Y. Yoo, Performance improvement of IPMC (Ionic Polymer Metal Composites) for a flapping actuator, Int. J. Control Autom. 4 (2006) 748-755.

[11] Y. Bar-Cohen, Electroactive polymers as artificial muscles: capabilities, potentials and challenges, Handbook on biomimetics, NTS Inc., Aug. 2001, pp.134.

[12] R. Pelrine, R. Kornbluh, Q. Pei, S. Stanford, S. Oh, and J. Eckerle, Dielectric elastomer artificial muscle actuators: toward biomimetic motion, Smart Structures and Materials 2002: Electroactive Polymer Actuators and Devices, Y. Bar-Cohen, Editor, in: Proceedings of SPIE, San Diego, 2002, pp. 126-137.

[13] Y. Liu, L. Liu, Z. Zhang and J. Leng, Dielectric elastomer film actuators: characterization, experiment and analysis, Smart Mater. Struct. 18 (2009) 095024.

[14] Md. Zulhash Uddin, M. Watanabe, H. Shirai, and T. Hirai, Creeping and novel huge bending of plasticized PVC, J. Robotics and Mechatronics, 14 (2002) 161-166.

[15] M. Yamano, N. Ogawa, M. Hashimoto, M. Takasaki and T.Hirai, A Contraction Type Soft Actuator Using Poly Vinyl Chloride Gel, in: Proceedings of IEEE International Conference on Robotics and Biomimetics (ROBIO 2008), Bangkok, Thailand, 2009, pp.745-750.

[16] N. Ogawa, M. Hashimoto, M. Takasaki and T. Hirai, Characteristics Evaluation of PVC Gel Actuators, in: The 2009 IEEE/RSJ Int. Conf. on Intelligent Robots and Systems (IROS2009), St. Louis, USA, 2009, pp.2898-2903.

[17] N. Hayasaka, S. Mao, Y. Tsuchiya and M. Hashimoto, Structural improvement and performance of thin-type PVC gel actuators, in: The JSME conference on Robotics and Mechatronics, 2013, pp. 2P1-F09. (in japanese)

[18] M. Shibagaki, N. Ogawa, M. Hashimoto and T. Hirai, Modeling of contraction type PVC gel actuators, in: Proc. of the 2010 IEEE Int. Conf. Robotics and Biomimetics (ROBIO 2010), Tianjin, China, 2010, pp.1434-1439.

[19] M. Shibagaki,T. Matsuki and M. Hashimoto, Application of a contraction type PVC gel actuator to brakes, in: Proc. of the 2010 IEEE Int. Conf. on Mechatronics and Automation (ICMA), Xian, China, 2010, pp.39-44.

[20] Y. Maeda, Y. Li, K. Yasuda and M. Hashimoto, Development of variable stiffness gel spats for walking assistance, in: The 2013 IEEE/RSJ Int. Conf. on Intelligent Robots and Systems (IROS2013), Tokyo, Japan, 2013, pp.5404-5409.

[21] H. Xia, M. Takasaki and T. Hirai, Actuation mechanism of plasticized PVC by electric field, Sens. Actuators A 157 (2010) 307-312.

[22] H. Xia, T. Ueki and and T. Hirai, Electrical response and mechanical behavior of plasticized PVC actuators, Adv. Mater. Res. 79-82 (2009) 2063-2066.

[23] K. Yasuda and M. Hashimoto, Simulation of electroactive PVC gel using FEM, in: The JSME conference on Robotics and Mechatronics, 2014, pp. 2A1-J05. (in japanese)

[24] J. M. Hollerbach, I. W. Hunter, J. Ballantyne, A comparative analysis of actuator technologies for robotics, in: The Robotics Review, MIT Press, Cambridge, MA, 1992, pp.299-342.

[25] R. H. Baughman, Playing nature's game with artificial muscles, Sci. 308 (2005) 63-65.

[26] B, K, Woods, M. F. Gentry, C. S. Kothera and N. M. Wereley, Fatigue life testing of swaged pneumatic artificial muscles as actuators for aerospace applications, J. Intel. Mat. Syst. Str. 23 (2012) 327-343
[27] C. S. Haines, M. D. Lima, N. Li, G. M. Spinks, J. Foroughi, J. D W.Madden, S. H. Kim, S. Fang, M. Jung de Andrade, F. G*oktepe, O. G“oktepe, S. M. Mirvakili, S. Naficy, X. Lepr`o, J. Oh, M. E. Kozlov, S. J. Kim, X. Xu, B. J. Swedlove, G. G. Wallace, and R. H. Baughman, Artificial muscles from fishing line and sewing thread, Sci. 343 (2014) 868-872.

[28] J. D. Madden, N. A. Vandesteeg, P. A. Anquetil, P. G. Madden, A. Takshi, R. Z. Pytel, and I. W. Hunter, Artificial muscle technology: Physical principles and naval prospects, IEEE J. Ocean. Eng. 29 (2004) 706-728.

[29] E. Malone and H. Lipson, Freeform fabrication of ionomeric polymer-metal composite actuators, Rapid Prototyping J. 12/5 (2006) 244-253.

[30] N. Ogawa, M. Hashimoto, M. Takasaki and T. Hirai, Development of an artificial muscle using PVC-gel, -Increasing force generation during the contraction-, in: The JSME conference on Robotics and Mechatronics, 2009, pp. 1A2-M03. (in japanese)

[31] A. V. Hill, The heat of shortening and the dynamic constants of muscle, Proceedings of the Royal Society B, London, 126 (1938) 136-195.

[32] B. Wohlfart, K. A. P. Edman, Rectangular hyperbola fitted to muscle force-velocity data using three-dimensional regression analysis, Exp. Physiol. 79 (1994) 235-239. 